Bangladesh J. Bot. 43(1): 1-8, 2014 (June)

\title{
COMPARATIVE STUDY OF FLORAL CHARACTERISTICS IN THE COMPONENT LINES OF HYBRID RICE (ORYZA SATIVA L.)
}

\author{
MJ Hasan*, Umma Kulsum, MH Rahman, A AKTer \\ AND AKM SHAMSUDDIN ${ }^{1}$ \\ Plant Breeding Division, Bangladesh Rice Research Institute, Gazipur-1701, Bangladesh
}

Key words: Floral characteristics, Component lines, Hybrid rice

\begin{abstract}
Significant variations were obtained among the component lines for most of the floral traits. BRRI9A exhibited superiority for angle of open florets, stigma length and breadth, anther breadth, filament length, style length and panicle exertion rate while its corresponding maintainer lines BRRI9B showed superiority for duration of open florets, stigma length and breadth, anther breadth and filament length. This indicated BRRI9A and its corresponding maintainer line BRRI9B possessed very good floral traits that influence out crossing. Among the restorer lines BR168R showed superiority for duration of open florets, angle of open florets, duration of bloomed florets/panicle, stigma length, anther length and number of pollen/microscopic focus. This suggested BR168R could be used as promising restorer line with BRRI9A for hybrid seed production in local condition.
\end{abstract}

\section{Introduction}

Bangladesh is the fourth largest producer and consumer of rice in the world with an annual production ranging from 21 to 22 million tons. About $75 \%$ of the total cropped land is covered by rice and more than $60 \%$ of the labor force is engaged in rice production. Rice alone contributes around $10 \%$ to the GDP. Thus the single crop rice has a multiple effect day to day daily life and economy (Iftekharuddaula et al. 2011).

One hundred and eight hybrid rice varieties so far released from National Seed Board under various private companies and public organization (SCA 2012). Majority of these hybrids are imported from China. In Boro seasons 2011 - 2012, total sold rice hybrid was 8800 MT of which 45\% were imported from China and the rest 55\% from local production (Huda and Ali 2012). If parental lines of those hybrids are locally adapted and adequate knowledge of floral morphology of parental lines could lead self sufficiency in $\mathrm{F}_{1}$ seed production. So, the knowledge of floral traits of A, B and R lines is very much important. There is ample scope to study the floral characteristics influencing out crossing rate of component lines of hybrid rice under local environment. It would help designing row ratio estimation for CMS multiplication and $\mathrm{F}_{1}$ seed production and ultimately helps in building cost effective seed production package development. With this view, this study was undertaken to compare the floral characteristics of the component lines of hybrid rice.

\section{Materials and Methods}

The experiment was conducted at the experimental field of Bangladesh Rice Research Institute (BRRI), Gazipur during T. Aman season 2007. Seven CMS lines, their corresponding maintainer lines and 30 restorer lines were evaluated to identify promising parental lines in respect of floral traits for developing heterotic rice hybrids with effective seed production

*Author for correspondence. <jamilbrri@yahoo.com>. ${ }^{1}$ Department of Genetics and Plant Breeding, Bangladesh Agricultural University, Mymensingh, Bangladesh. 
potentiality. There were three blocks in the experimental field: one for CMS, another for maintainer and the other for restorer lines. Seedlings of each of these lines were raised in bed and 21 days old seedlings were transplanted in one plant/hill in the experimental plots. The plots were 3.5 meter in length containing three rows. The plant spacing provided was $20 \mathrm{~cm}$ between rows and $15 \mathrm{~cm}$ between plants of the same row. Adequate soil fertility was ensured by applying ureaTSP-MP-gypsum-ZnSO $@$ @ 150:100:70:60:10 kg/ha, respectively. Total TSP, MP, gypsum and $\mathrm{ZnSO}_{4}$ were applied during final land preparation.

The urea was applied in three installments, at 15 days after transplanting (DAT), 30 and 45 DAT. Necessary intercultural operations were carried out during cropping period for proper growth and development of the plants. Five sample plants were randomly selected from each plot excluding the border plants and the following data were recorded: duration of floret open (DOF), per cent panicle exertion (PPE), angle of open floret (AOF), stigma length (SL), stigma breadth (SB), anther length (AL), anther breadth (AB), stigma exertion rate (SER), filament length (FL), out crossing rate (OCR), days to $50 \%$ flowering and days to maturity. Maintainer and restorer lines are inbred lines so, panicle exertion, stigma exertion and out crossing rate were not measured for them. Number of pollen/microscopic focus was measured only for restorer line. Data were analyzed according to MSTAT C software.

\section{Results and Discussion}

Analyses of variance showed the presence of significant variation among the tested CMS lines in respect of most the floral characters except for stigma and style length at $1 \%$ level of probability. While the corresponding maintainer lines showed non-significant variation for panicle emergence, duration of bloomed florets/panicle and style length. Restorer lines showed significant variation for most of the characters except for angle of open floret, stigma and anther breadth (Table 1). CMS lines and its corresponding maintainer lines are genetically isogenic except for pollen fertility. Maintainer lines showed viable pollen and it maintained the sterility character of corresponding CMS lines. Mean, range, standard deviation and coefficient of variation of the studied component lines are presented in Table 2. Duration of open florets was high for CMS lines and it ranged from 130 to 254 min with an average of 194.27 min. The CMS line Jin23A was found to remain open for longest period $(254 \mathrm{~min})$ and the shortest period of opening of florets (130 min) was noticed in D.ShanA (Table 3).

Table 1. Analysis of variance for floral characters of the CMS, maintainer and restorer lines grown in T. Aman season of 2007.

\begin{tabular}{lccccccc}
\hline $\begin{array}{l}\text { Sources } \\
\text { of } \\
\text { variation }\end{array}$ & $\begin{array}{c}\text { D.f } \\
\text { Replication (A line) }\end{array}$ & $\begin{array}{c}\text { Duration of } \\
\text { open florets } \\
(\mathrm{min})\end{array}$ & $\begin{array}{c}\text { Angle of } \\
\text { open floret } \\
\left(\mathrm{O}^{\circ}\right)\end{array}$ & $\begin{array}{c}\text { Panicle } \\
\text { emergence } \\
\text { (days) }\end{array}$ & $\begin{array}{c}\text { Duration of } \\
\text { bloomed florets/ } \\
\text { panicle (days) }\end{array}$ & $\begin{array}{c}\text { Stigma } \\
\text { length } \\
(\mathrm{mm})\end{array}$ & $\begin{array}{c}\text { Stigma } \\
\text { breadth } \\
(\mathrm{mm})\end{array}$ \\
\hline Replication (B line) & 2 & 8.714 & 0.360 & 0.571 & 3.571 & 0.061 & 0.005 \\
Replication (R line) & 2 & 44.878 & 0.241 & 1.000 & 2.286 & 0.088 & 0.012 \\
Genotype (A line) & 6 & $6236.714^{* *}$ & $22.000^{* *}$ & $2.429^{* *}$ & $3.429^{* *}$ & 0.441 & 0.034 \\
Genotype (B line) & 6 & $149.714^{* *}$ & $10.107^{* *}$ & $1.000 \mathrm{~ns}$ & $1.429 \mathrm{~ns}$ & $0.1155^{* *}$ & $0.037^{* *}$ \\
Genotype (R line) & 29 & $43.390^{* *}$ & $5.324 \mathrm{~ns}$ & $1.848 * *$ & $2.307^{* *}$ & $0.085^{* *}$ & $0.015 \mathrm{~ns}$ \\
Error (A line) & 12 & 28.714 & 0.960 & 0.405 & 0.571 & 0.038 & 0.002 \\
Error (B line) & 12 & 6.214 & 0.805 & 0.500 & 0.786 & 0.019 & 0.002 \\
Error (R line) & 58 & 3.981 & 4.185 & 0.541 & 0.493 & 0.031 & 0.008 \\
\hline
\end{tabular}

(Contd.) 
Table contd. from right hand side.

\begin{tabular}{llllccccl}
\hline $\begin{array}{c}\text { Anther } \\
\text { length } \\
(\mathrm{mm})\end{array}$ & $\begin{array}{c}\text { Anther } \\
\text { breadth } \\
(\mathrm{mm})\end{array}$ & $\begin{array}{c}\text { Filament } \\
\text { length } \\
(\mathrm{mm})\end{array}$ & $\begin{array}{c}\text { Style } \\
\text { length } \\
(\mathrm{mm})\end{array}$ & $\begin{array}{c}\text { Panicle } \\
\text { exertio } \\
\text { n rate } \\
(\%)\end{array}$ & $\begin{array}{c}\text { Stigma } \\
\text { exertion } \\
\text { rate }(\%)\end{array}$ & $\begin{array}{c}\text { Out } \\
\text { crossing } \\
\text { rate (\%) }\end{array}$ & $\begin{array}{c}\text { Days 50\% } \\
\text { flowering }\end{array}$ & $\begin{array}{c}\text { Days to } \\
\text { maturity }\end{array}$ \\
\hline 0.019 & 0.000 & 0.046 & 0.036 & 9.903 & 15.168 & 0.124 & 35.286 & 8.333 \\
0.036 & 0.001 & 0.096 & 0.022 & - & - & - & 21.143 & 7.00 \\
0.156 & 0.005 & 0.224 & 0.116 & - & - & - & 22.411 & 48.633 \\
$0.238^{* *}$ & $0.008^{* *}$ & $1.530^{* *}$ & $0.045 \mathrm{~ns}$ & $116.834^{* *}$ & $291.568^{* *}$ & $2.103^{* *}$ & $218.714^{* *}$ & $179.048^{* *}$ \\
$0.087^{* *}$ & $0.013^{* *}$ & $1.009^{* *}$ & $0.020 \mathrm{~ns}$ & - & - & - & $199.714^{* *}$ & $178.00^{* *}$ \\
$0.282^{* *}$ & $0.022 \mathrm{~ns}$ & $0.590^{* *}$ & $0.093^{* *}$ & - & - & - & $195.975^{* *}$ & $249.590^{* *}$ \\
0.028 & 0.001 & 0.033 & 0.017 & 10.395 & 9.870 & 0.119 & 7.619 & 11.667 \\
0.013 & 0.001 & 0.107 & 0.010 & - & - & - & 7.310 & 9.167 \\
0.077 & 0.013 & 0.160 & 0.034 & - & - & - & 14.377 & 7.357 \\
\hline
\end{tabular}

Table 2. Mean, range, standard deviation (SD) and coefficient of variation (CV) for floral characters of the CMS, maintainer and restorer lines grown in T. Aman season of 2007.

\begin{tabular}{llllllll}
\hline $\begin{array}{l}\text { Sources } \\
\text { of } \\
\text { variation }\end{array}$ & $\begin{array}{c}\text { Duration of } \\
\text { open florets } \\
(\mathrm{min})\end{array}$ & $\begin{array}{c}\text { Angle of } \\
\text { open floret } \\
\left(\mathrm{O}^{\circ}\right)\end{array}$ & $\begin{array}{c}\text { Panicle } \\
\text { emergence } \\
\text { (days) }\end{array}$ & $\begin{array}{c}\text { Duration of } \\
\text { bloomed florets/ } \\
\text { panicle (days) }\end{array}$ & $\begin{array}{c}\text { Stigma } \\
\text { length } \\
(\mathrm{mm})\end{array}$ & $\begin{array}{c}\text { Stigma } \\
\text { breadth } \\
(\mathrm{mm})\end{array}$ & $\begin{array}{c}\text { Anther } \\
\text { length } \\
(\mathrm{mm})\end{array}$ \\
\hline Mean (A line) & 194.29 & 24.00 & 4.86 & 5.86 & 1.09 & 0.38 & 2.20 \\
Mean (B line) & 62.05 & 21.93 & 3.00 & 3.86 & 0.88 & 0.32 & 1.97 \\
Mean (R line) & 36.20 & 21.60 & 4.37 & 4.60 & 0.86 & 0.30 & 2.39 \\
Range (A line) & $130-254$ & $20-27$ & $4.0-6.0$ & $4.0-7.0$ & $1-1.40$ & $0.22-0.55$ & $2.00-2.70$ \\
Range (B line) & $54-71$ & $19.50-25.00$ & $2.0-4.0$ & $3.0-5.0$ & $0.60-1.25$ & $0.20-0.50$ & $1.8-2.3$ \\
Range (R line) & $30.0-42.0$ & $19.0-24.0$ & $3.0-6.0$ & $3.0-6.0$ & $0.60-1.20$ & $0.20-0.40$ & $1.90-3.00$ \\
SD (A line) & 43.47 & 2.69 & 1.01 & 1.32 & 0.22 & 0.13 & 0.30 \\
SD (B line) & 7.03 & 1.88 & 0.84 & 1.06 & 0.23 & 0.12 & 0.19 \\
SD (R line) & 4.197 & 2.283 & 0.964 & 1.070 & 0.236 & 0.103 & 0.334 \\
CV (\%)(A line) & 2.76 & 4.08 & 13.10 & 12.91 & 17.98 & 10.49 & 7.57 \\
CV (\%) (B line) & 4.02 & 4.09 & 23.57 & 22.98 & 15.71 & 14.34 & 5.75 \\
CV (\%) ( R line) & 5.74 & 9.50 & 17.24 & 16.33 & 20.04 & 32.60 & 11.87 \\
\hline
\end{tabular}

Table contd. from right hand side.

\begin{tabular}{|c|c|c|c|c|c|c|c|}
\hline $\begin{array}{l}\text { Anther } \\
\text { breadth } \\
(\mathrm{mm})\end{array}$ & $\begin{array}{c}\text { Filament } \\
\text { length } \\
(\mathrm{mm})\end{array}$ & $\begin{array}{c}\text { Style } \\
\text { length } \\
(\mathrm{mm})\end{array}$ & $\begin{array}{c}\text { Panicle } \\
\text { exertion rate } \\
(\%)\end{array}$ & $\begin{array}{l}\text { Stigma } \\
\text { exertion } \\
\text { rate (\%) }\end{array}$ & $\begin{array}{c}\text { Out } \\
\text { crossing } \\
\text { rate (\%) }\end{array}$ & $\begin{array}{c}\text { Days } \\
50 \% \\
\text { flowering }\end{array}$ & $\begin{array}{c}\text { Days } \\
\text { to } \\
\text { maturity }\end{array}$ \\
\hline 0.42 & 4.38 & 0.89 & 67.89 & 67.15 & 1.88 & 82.29 & 109.95 \\
\hline 0.49 & 4.64 & 0.70 & - & - & - & 78.71 & 107.00 \\
\hline 0.53 & 5.09 & 0.96 & - & - & - & 92.57 & 120.33 \\
\hline $0.35-0.50$ & $3.00-5.00$ & $0.70-1.00$ & 59.93-77.22 & $51.30-75.67$ & $1.06-3.33$ & $69-95$ & $97-121$ \\
\hline $0.40-0.60$ & $4.0-5.50$ & $0.60-0.80$ & - & - & - & $66.0-90.0$ & $94.0-119.0$ \\
\hline $0.40-0.70$ & $4.20-6.00$ & $0.60-1.20$ & - & - & - & 72.3-105.0 & $98.0-135.0$ \\
\hline 0.06 & 0.70 & 0.17 & 6.50 & 9.74 & 0.85 & 8.57 & 7.85 \\
\hline 0.07 & 0.61 & 0.12 & - & - & - & 8.15 & 7.72 \\
\hline 0.110 & 0.522 & 0.236 & - & - & - & 8.046 & 10.216 \\
\hline 7.80 & 4.15 & 14.85 & 4.75 & 4.68 & 18.35 & 3.35 & 3.11 \\
\hline 7.16 & 7.06 & 14.37 & - & - & - & 3.43 & 2.83 \\
\hline 22.20 & 8.00 & 19.22 & - & - & - & 4.14 & 2.28 \\
\hline
\end{tabular}

$\mathrm{B}$ and $\mathrm{R}$ lines are inbred lines so, panicle exertion, stigma exertion and out crossing rate were not measured. 
Table 3. Mean values of the floral characters of the CMS and maintainer lines grown in T. Aman season of 2007.

\begin{tabular}{|c|c|c|c|c|c|c|c|}
\hline Genotypes & $\begin{array}{l}\text { Duration of } \\
\text { open florets } \\
\text { (min) }\end{array}$ & $\begin{array}{l}\text { Angle of } \\
\text { open floret } \\
\left(\mathrm{O}^{\circ}\right)\end{array}$ & $\begin{array}{c}\text { Panicle } \\
\text { emergence } \\
\text { (days) }\end{array}$ & $\begin{array}{l}\text { Duration of bloomed } \\
\text { florets/panicle } \\
\text { (days) }\end{array}$ & $\begin{array}{l}\text { Stigma } \\
\text { length } \\
(\mathrm{mm})\end{array}$ & $\begin{array}{l}\text { Stigma } \\
\text { breadth } \\
(\mathrm{mm})\end{array}$ & $\begin{array}{l}\text { Anther } \\
\text { length } \\
(\mathrm{mm})\end{array}$ \\
\hline IR58025A & 181d & $20 \mathrm{c}$ & $5 \mathrm{~b}$ & $6 \mathrm{~b}$ & $1^{\mathrm{NS}}$ & $0.40 \mathrm{~b}$ & $2.10 \mathrm{c}$ \\
\hline IR58025B & $60 \mathrm{c}$ & $19.50 \mathrm{~d}$ & $4.00^{\mathrm{NS}}$ & $4.00^{\mathrm{NS}}$ & $0.80 \mathrm{~b}$ & $0.33 \mathrm{c}$ & $1.80 \mathrm{c}$ \\
\hline II32A & $200 \mathrm{c}$ & $26 \mathrm{a}$ & $6 \mathrm{a}$ & $7 \mathrm{a}$ & $1^{\mathrm{NS}}$ & $0.40 \mathrm{~b}$ & $2.00 \mathrm{c}$ \\
\hline II32B & $65 \mathrm{~b}$ & $25.00 \mathrm{a}$ & $2.00^{\mathrm{NS}}$ & $4.00^{\mathrm{NS}}$ & $0.90 \mathrm{~b}$ & $0.30 \mathrm{c}$ & $1.90 \mathrm{c}$ \\
\hline Jin23A & $254 \mathrm{a}$ & $27 \mathrm{a}$ & $5 \mathrm{~b}$ & $6 \mathrm{~b}$ & $1^{\mathrm{NS}}$ & $0.22 \mathrm{c}$ & $2.00 \mathrm{c}$ \\
\hline Jin23B & $58 \mathrm{~cd}$ & $23.00 \mathrm{~b}$ & $3.00^{\mathrm{NS}}$ & $3.00^{\mathrm{NS}}$ & $0.90 \mathrm{~b}$ & $0.20 \mathrm{~d}$ & $1.90 \mathrm{c}$ \\
\hline IR78362A & $150 \mathrm{e}$ & $23 \mathrm{~b}$ & $4 \mathrm{c}$ & $5 \mathrm{c}$ & $1^{\mathrm{NS}}$ & $0.22 \mathrm{c}$ & $2.05 \mathrm{c}$ \\
\hline IR78362B & $71 \mathrm{a}$ & $21.00 \mathrm{c}$ & $3.00^{\mathrm{NS}}$ & $3.00^{\mathrm{NS}}$ & $0.80 \mathrm{~b}$ & $0.20 \mathrm{~d}$ & $1.90 \mathrm{c}$ \\
\hline D.ShanA & $130 \mathrm{f}$ & $23 \mathrm{~b}$ & $6 \mathrm{a}$ & $7 \mathrm{a}$ & $1^{\mathrm{NS}}$ & $0.50 \mathrm{a}$ & $2.70 \mathrm{a}$ \\
\hline D.ShanB & $54 \mathrm{e}$ & $21.00 \mathrm{c}$ & $3.00^{\mathrm{NS}}$ & $5.00^{\mathrm{NS}}$ & $0.60 \mathrm{c}$ & $0.43 \mathrm{~b}$ & $2.30 \mathrm{a}$ \\
\hline Gan46A & $245 \mathrm{~b}$ & $22 \mathrm{~b}$ & $4 \mathrm{c}$ & $4 \mathrm{~d}$ & $1^{\mathrm{NS}}$ & $0.40 \mathrm{~b}$ & $2.05 \mathrm{c}$ \\
\hline Gan46B & 55.33 de & $21.00 \mathrm{c}$ & $3.00^{\mathrm{NS}}$ & $4.00^{\mathrm{NS}}$ & $0.90 \mathrm{~b}$ & $0.30 \mathrm{c}$ & $1.90 \mathrm{c}$ \\
\hline BRRI9A & $200 \mathrm{c}$ & $27 \mathrm{a}$ & $4 \mathrm{c}$ & $6 \mathrm{~b}$ & $1.4^{\mathrm{NS}}$ & 0.55 a & $2.50 \mathrm{~b}$ \\
\hline BRRI9B & $71 \mathrm{a}$ & $23.00 \mathrm{~b}$ & $3.00^{\mathrm{NS}}$ & $4.00^{\mathrm{NS}}$ & $1.25 \mathrm{a}$ & $0.50 \mathrm{a}$ & $2.10 \mathrm{~b}$ \\
\hline
\end{tabular}

Table contd. from right hand side

\begin{tabular}{|c|c|c|c|c|c|c|c|}
\hline $\begin{array}{l}\text { Anther } \\
\text { breadth } \\
(\mathrm{mm})\end{array}$ & $\begin{array}{c}\text { Filament } \\
\text { length } \\
(\mathrm{mm})\end{array}$ & $\begin{array}{l}\text { Style length } \\
\text { (mm) }\end{array}$ & $\begin{array}{c}\text { Panicle } \\
\text { exertion rate } \\
(\%)\end{array}$ & $\begin{array}{l}\text { Stigma } \\
\text { exertion } \\
\text { rate (\%) }\end{array}$ & $\begin{array}{l}\text { Out crossing } \\
\text { rate } \\
(\%)\end{array}$ & $\begin{array}{c}\text { Days to } \\
50 \% \\
\text { flowering }\end{array}$ & $\begin{array}{l}\text { Days to } \\
\text { maturity }\end{array}$ \\
\hline $0.40 \mathrm{~b}$ & $4.87 \mathrm{ab}$ & $0.70^{\mathrm{NS}}$ & $59.93 \mathrm{e}$ & $70.51 \mathrm{c}$ & $3.333 \mathrm{a}$ & $86 \mathrm{~b}$ & $114 \mathrm{~b}$ \\
\hline $0.50 \mathrm{~b}$ & $5.00 \mathrm{~b}$ & $0.60^{\mathrm{NS}}$ & - & - & - & $83.00 \mathrm{~b}$ & 110.0 bc \\
\hline $0.40 \mathrm{~b}$ & $4.60 \mathrm{c}$ & $1.00^{\mathrm{NS}}$ & $64.73 \mathrm{~cd}$ & $51.30 \mathrm{~d}$ & $1.207 \mathrm{~d}$ & 81 c & $110 \mathrm{c}$ \\
\hline $0.50 \mathrm{~b}$ & $4.90 \mathrm{~b}$ & $0.70^{\mathrm{NS}}$ & - & - & - & $77.00 \mathrm{c}$ & $107.0 \mathrm{~cd}$ \\
\hline 0.35 c & $3.80 \mathrm{~d}$ & $0.90^{\mathrm{NS}}$ & 62.36 de & 72.44 abc & $2.230 \mathrm{~b}$ & $77 \mathrm{~d}$ & $106 \mathrm{c}$ \\
\hline $0.40 \mathrm{c}$ & $4.00 \mathrm{c}$ & $0.80^{\mathrm{NS}}$ & - & - & - & $74.00 \mathrm{c}$ & $104.0 \mathrm{~d}$ \\
\hline $0.40 \mathrm{~b}$ & $3.00 \mathrm{e}$ & $0.80^{\mathrm{NS}}$ & $73.25 \mathrm{~b}$ & $75.67 \mathrm{a}$ & $1.063 \mathrm{~d}$ & 79 cd & 106.7 c \\
\hline 0.42 c & $4.00 \mathrm{c}$ & $0.70^{\mathrm{NS}}$ & - & - & - & $75.00 \mathrm{c}$ & $104.0 \mathrm{~d}$ \\
\hline $0.40 \mathrm{~b}$ & 4.70 bc & $0.79^{\mathrm{NS}}$ & $66.40 \mathrm{c}$ & 70.80 bc & $1.150 \mathrm{~d}$ & $69 \mathrm{e}$ & $97.00 \mathrm{~d}$ \\
\hline $0.50 \mathrm{~b}$ & 4.20 c & $0.70^{\mathrm{NS}}$ & - & - & - & $66.00 \mathrm{~d}$ & $94.00 \mathrm{e}$ \\
\hline $0.48 \mathrm{a}$ & $4.60 \mathrm{c}$ & $1.00^{\mathrm{NS}}$ & $71.30 \mathrm{~b}$ & $54.80 \mathrm{~d}$ & $1.767 \mathrm{c}$ & 95 a & $121.0 \mathrm{a}$ \\
\hline $0.50 \mathrm{~b}$ & $4.90 \mathrm{~b}$ & $0.80^{\mathrm{NS}}$ & - & - & - & $90.00 \mathrm{a}$ & $119.0 \mathrm{a}$ \\
\hline $0.50 \mathrm{a}$ & $5.00 \mathrm{a}$ & $1.00^{\mathrm{NS}}$ & $77.22 \mathrm{a}$ & $74.51 \mathrm{ab}$ & $2.433 \mathrm{~b}$ & 89 b & $115.0 \mathrm{~b}$ \\
\hline $0.60 \mathrm{a}$ & $5.50 \mathrm{a}$ & $0.60^{\mathrm{NS}}$ & - & - & - & $86.00 \mathrm{~b}$ & $111.0 \mathrm{~b}$ \\
\hline
\end{tabular}

Values with same letter(s) are statistically identical at 5\% level of probability. Maintainer line is inbred line so panicle exertion, stigma exertion and out crossing rate was not measured.

Maintainer lines remained open less than their corresponding CMS lines and it ranged from 54 to 71 min with a mean value of $62.05 \mathrm{~min}$. Restorer lines florets remained open for shorter than CMS and maintainer lines and it ranged from 30 to $42 \mathrm{~min}$ with a mean value of $36.20 \mathrm{~min}$ (Table 2). Angle of open floret was the highest for BRRI9A and Jin23A and the lowest for IR58025A and it ranged from 20 to 27 degree with an average of 24 degree (Tables 2 and 3). A wide in male sterile plant is desirable because it would help cross-pollination (Virmani and Edwards 1983). Maintainer and restorer lines showed less floret opening angle. Duration of bloomed florets was found to vary significantly among the tested A and B and R lines (Table 1). Stigma length is an important trait for CMS line because long stigma facilitates out crossing rate of a CMS line. 
Stigma length did not vary significantly and it ranged from 1 to $1.4 \mathrm{~mm}$. The highest stigma length was recorded in CMS line BRRI9A $(1.4 \mathrm{~mm})$ and the rest showed similar length. Oka (1988) stated that stigma length $(>1 \mathrm{~mm})$ had a pronounced influence on out crossing rate of a CMS line. Stigma breadth varied significantly among the CMS lines and recorded highest in BRRI9A $(0.55 \mathrm{~mm})$ and lowest in Jin23A and IR78362A $(0.22 \mathrm{~mm})$. Stigma length and breadth varied significantly among maintainer lines. The maximum length of stigma was recorded in BRRI9B $(1.25 \mathrm{~mm})$ and the minimum was in D.ShanB $(0.60 \mathrm{~mm})$ (Table 3). Stigma length varied significantly among restorer lines and the highest was recorded in BR168R $(1.2 \mathrm{~mm})$ along with IR73004-7-3-3-3R and IR73013-95-1-3-2R (Table 4) but breadth was not varied significantly. Anther length for CMS lines ranged from 2 to $2.70 \mathrm{~mm}$ with an average of $2.20 \mathrm{~mm}$. The lowest anther length was recorded in Jin23A and II32A $(2 \mathrm{~mm})$ and the highest was in D.ShanA (2.7 $\mathrm{mm}$ ) Variation in anther breadth was significant among the tested CMS lines. Anther length for maintainer lines ranged from $1.8 \mathrm{~mm}$ to $2.3 \mathrm{~mm}$.

Table 4. Mean values of floral traits of restorer lines grown in T. Aman season of 2007.

\begin{tabular}{|c|c|c|c|c|c|}
\hline Genotypes & $\begin{array}{l}\text { Duration of } \\
\text { open florets } \\
\text { (min) }\end{array}$ & $\begin{array}{c}\text { Angle of } \\
\text { open floret } \\
\left(\mathrm{O}^{\circ}\right)\end{array}$ & $\begin{array}{c}\text { Panicle } \\
\text { emergence } \\
\text { (days) }\end{array}$ & $\begin{array}{c}\text { Duration of } \\
\text { bloomed florets/ } \\
\text { panicle(days) }\end{array}$ & $\begin{array}{c}\text { Stigma } \\
\text { length } \\
(\mathrm{mm})\end{array}$ \\
\hline BR827R & $39.00 \mathrm{~cd}$ & $19.00^{\text {NS }}$ & $4.00 \mathrm{c}$ & $5.00 \mathrm{~b}$ & $0.90 \mathrm{~cd}$ \\
\hline BR168R & $42.00 \mathrm{a}$ & $24.00^{\mathrm{NS}}$ & $5.00 \mathrm{~b}$ & $6.00 \mathrm{a}$ & $1.20 \mathrm{a}$ \\
\hline BR736R & 38.00 de & $21.00^{\mathrm{NS}}$ & $5.00 \mathrm{~b}$ & $5.00 \mathrm{~b}$ & 0.70 ef \\
\hline BR6723-1-1-2R & $31.00 \mathrm{ij}$ & $23.00^{\mathrm{Ns}}$ & $3.00 \mathrm{~d}$ & $4.00 \mathrm{c}$ & 0.80 de \\
\hline BR6839-41-5-1R & $37.00 \mathrm{e}$ & $22.00^{\text {NS }}$ & $3.00 \mathrm{~d}$ & $5.00 \mathrm{~b}$ & $0.80 \mathrm{e}$ \\
\hline BR7013-62-1-1R & $40.00 \mathrm{bc}$ & $20.00^{\text {NS }}$ & $4.00 \mathrm{c}$ & $5.00 \mathrm{~b}$ & 0.70 ef \\
\hline BR7011-37-1-2R & $40.00 \mathrm{bc}$ & $22.00^{\mathrm{NS}}$ & $5.00 \mathrm{~b}$ & $6.00 \mathrm{a}$ & 0.70 ef \\
\hline M.H.63R & $35.00 \mathrm{f}$ & $22.00^{\text {NS }}$ & $3.00 \mathrm{~d}$ & $3.00 \mathrm{~d}$ & $1.00 \mathrm{bc}$ \\
\hline M.H77R & $37.00 \mathrm{e}$ & $21.00^{\mathrm{NS}}$ & $3.00 \mathrm{~d}$ & $4.00 \mathrm{c}$ & $0.90 \mathrm{~cd}$ \\
\hline Gui 99R & $30.00 \mathrm{j}$ & $23.00^{\mathrm{NS}}$ & $4.00 \mathrm{c}$ & $4.00 \mathrm{c}$ & $1.10 \mathrm{ab}$ \\
\hline Wan3R & $32.00 \mathrm{hi}$ & $21.00^{\mathrm{NS}}$ & $4.00 \mathrm{c}$ & $3.00 \mathrm{~d}$ & $0.80 \mathrm{de}$ \\
\hline IR40750R & $41.00 \mathrm{ab}$ & $20.00^{\text {NS }}$ & $5.00 \mathrm{~b}$ & $3.00 \mathrm{~d}$ & $1.00 \mathrm{c}$ \\
\hline IR7320-44-2-3-IR & $35.00 \mathrm{f}$ & $21.00^{\mathrm{NS}}$ & $4.00 \mathrm{c}$ & $4.00 \mathrm{c}$ & $0.75 \mathrm{e}$ \\
\hline PSBRC82R & $41.00 \mathrm{ab}$ & $24.00^{\mathrm{NS}}$ & $6.00 \mathrm{a}$ & $6.00 \mathrm{a}$ & $1.20 \mathrm{a}$ \\
\hline IR68011-15-1-12-3R & $35.00 \mathrm{f}$ & $22.00^{\mathrm{NS}}$ & $5.00 \mathrm{~b}$ & $4.00 \mathrm{c}$ & 0.80 de \\
\hline IR73004-107-3-3-2R & $33.00 \mathrm{gh}$ & $22.00^{\text {NS }}$ & $4.00 \mathrm{c}$ & $5.00 \mathrm{~b}$ & $1.00 \mathrm{c}$ \\
\hline IR73004-7-3-3-3R & $39.67 \mathrm{c}$ & $21.00^{\mathrm{NS}}$ & $4.00 \mathrm{c}$ & $5.00 \mathrm{~b}$ & $1.20 \mathrm{a}$ \\
\hline IR64R & 32.00 hi & $23.00^{\mathrm{NS}}$ & $4.00 \mathrm{c}$ & $4.00 \mathrm{c}$ & $1.00 \mathrm{bc}$ \\
\hline IR46R & $30.00 \mathrm{j}$ & $21.00^{\text {NS }}$ & $4.00 \mathrm{c}$ & $4.00 \mathrm{c}$ & 0.80 de \\
\hline IR71137-328-2-3-3-3-2R & $39.00 \mathrm{~cd}$ & $24.00^{\text {NS }}$ & $4.00 \mathrm{c}$ & $5.00 \mathrm{~b}$ & 0.70 ef \\
\hline IR 73885-1-4-1-4-4-3-6R & $30.00 \mathrm{j}$ & $22.00^{\mathrm{NS}}$ & $4.00 \mathrm{c}$ & $5.00 \mathrm{~b}$ & $1.00 \mathrm{bc}$ \\
\hline IR69716-37-1-1-5-IR & 32.00 hi & $21.00^{\mathrm{NS}}$ & $5.00 \mathrm{~b}$ & $5.00 \mathrm{~b}$ & 0.80 de \\
\hline AjayaR & $33.00 \mathrm{gh}$ & $20.00^{\text {NS }}$ & $5.00 \mathrm{~b}$ & $5.00 \mathrm{~b}$ & 0.80 de \\
\hline IR73013-95-1-3-2R & $34.00 \mathrm{fg}$ & $22.00^{\mathrm{NS}}$ & $5.00 \mathrm{~b}$ & $4.00 \mathrm{c}$ & $1.20 \mathrm{a}$ \\
\hline IR44675R & $30.00 \mathrm{j}$ & $19.00^{\mathrm{NS}}$ & $5.00 \mathrm{~b}$ & $3.00 \mathrm{~d}$ & $0.60 \mathrm{f}$ \\
\hline IR32809-26-3-3R & $32.00 \mathrm{hi}$ & $23.00^{\mathrm{Ns}}$ & $4.00 \mathrm{c}$ & $4.00 \mathrm{c}$ & $1.10 \mathrm{~b}$ \\
\hline IR72887-38-1-3-2R & 32.00 hi & $22.00^{\mathrm{NS}}$ & $3.00 \mathrm{~d}$ & $4.00 \mathrm{c}$ & 0.80 de \\
\hline IR72906-24-1-3-IR & $31.00 \mathrm{ij}$ & $20.00^{\mathrm{NS}}$ & $5.00 \mathrm{~b}$ & $3.00 \mathrm{~d}$ & $0.90 \mathrm{~cd}$ \\
\hline IR69702-3-2-3R & $33.00 \mathrm{gh}$ & $20.00^{\text {NS }}$ & $5.00 \mathrm{~b}$ & $3.00 \mathrm{~d}$ & 0.70 ef \\
\hline IR69701-41-3-IR & $39.00 \mathrm{~cd}$ & $23.00^{\text {NS }}$ & $5.00 \mathrm{~b}$ & $4.00 \mathrm{c}$ & 0.70 ef \\
\hline
\end{tabular}


Table contd. from right hand side

\begin{tabular}{|c|c|c|c|c|c|c|c|}
\hline $\begin{array}{l}\text { Stigma } \\
\text { breadth } \\
(\mathrm{mm})\end{array}$ & $\begin{array}{l}\text { Anther } \\
\text { length } \\
(\mathrm{mm})\end{array}$ & $\begin{array}{c}\text { Anther } \\
\text { breadth } \\
(\mathrm{mm})\end{array}$ & $\begin{array}{c}\text { Filament } \\
\text { length } \\
(\mathrm{mm})\end{array}$ & $\begin{array}{l}\text { Style } \\
\text { length } \\
(\mathrm{mm})\end{array}$ & $\begin{array}{l}\text { Days } 50 \% \\
\text { flowering }\end{array}$ & $\begin{array}{l}\text { No. of pollen/ } \\
\text { microscopic } \\
\text { focus }\end{array}$ & $\begin{array}{l}\text { Days to } \\
\text { maturity }\end{array}$ \\
\hline $0.24^{\mathrm{NS}}$ & $2.10 \mathrm{jk}$ & $0.60^{\mathrm{NS}}$ & 5.50 bc & 0.70 ef & 100.0 bc & $1250.0 \mathrm{i}$ & 130.0 c \\
\hline $0.35^{\mathrm{NS}}$ & $3.00 \mathrm{a}$ & $0.60^{\mathrm{NS}}$ & $5.60 \mathrm{~b}$ & 0.70 ef & 100.0 bc & $2130.0 \mathrm{a}$ & $129.0 \mathrm{c}$ \\
\hline $0.26^{\mathrm{NS}}$ & $2.00 \mathrm{kl}$ & $0.50^{\mathrm{NS}}$ & 5.30 cde & $0.60 \mathrm{f}$ & $101.0 \mathrm{~b}$ & $1410.0 \mathrm{~g}$ & $130.0 \mathrm{c}$ \\
\hline $0.40^{\mathrm{NS}}$ & $2.20 \mathrm{ij}$ & $0.60^{\mathrm{NS}}$ & $4.80 \mathrm{hi}$ & 0.80 de & $81.00 \mathrm{ij}$ & $2150.0 \mathrm{a}$ & 107.01 \\
\hline $0.23^{\mathrm{NS}}$ & $2.10 \mathrm{jk}$ & $0.50^{\text {NS }}$ & $4.50 \mathrm{jk}$ & $1.00 \mathrm{bc}$ & $89.00 \mathrm{~g}$ & $2117.0 \mathrm{a}$ & $118.0 \mathrm{fg}$ \\
\hline $0.20^{\mathrm{NS}}$ & $2.20 \mathrm{ij}$ & $0.46^{\mathrm{NS}}$ & 4.90 ghi & $1.00 \mathrm{bc}$ & $90.00 \mathrm{fg}$ & $1900.0 \mathrm{c}$ & $118.0 \mathrm{fg}$ \\
\hline $0.23^{\text {NS }}$ & $2.30 \mathrm{hi}$ & $0.60^{\mathrm{NS}}$ & 4.80 hi & $1.00 \mathrm{bc}$ & $90.00 \mathrm{~g}$ & $1800.0 \mathrm{~d}$ & $119.0 \mathrm{f}$ \\
\hline $0.26^{\mathrm{NS}}$ & $3.00 \mathrm{a}$ & $0.60^{\mathrm{NS}}$ & $5.00 \mathrm{fgh}$ & $1.00 \mathrm{bc}$ & $82.00 \mathrm{ij}$ & $300.0 \mathrm{t}$ & $109.0 \mathrm{jk}$ \\
\hline $0.20^{\mathrm{NS}}$ & $2.10 \mathrm{jk}$ & $0.45^{\mathrm{NS}}$ & $4.70 \mathrm{ij}$ & $1.10 \mathrm{ab}$ & $82.00 \mathrm{ij}$ & $690.0 \mathrm{p}$ & $110.0 \mathrm{ij}$ \\
\hline $0.29^{\mathrm{NS}}$ & $2.20 \mathrm{ij}$ & $0.42^{\mathrm{NS}}$ & 4.90 ghi & 0.70 ef & $81.00 \mathrm{ij}$ & $853.0 \mathrm{n}$ & $108.0 \mathrm{kl}$ \\
\hline $0.40^{\mathrm{NS}}$ & $3.00 \mathrm{a}$ & $0.50^{\mathrm{NS}}$ & 5.40 bcd & $1.00 \mathrm{bc}$ & 80.00 j & $2000.0 \mathrm{~b}$ & 107.01 \\
\hline $0.32^{\text {NS }}$ & $2.20 \mathrm{ij}$ & $0.54^{\text {NS }}$ & 5.30 cde & $1.20 \mathrm{a}$ & 94.00 de & $850.0 \mathrm{n}$ & $122.0 \mathrm{e}$ \\
\hline $0.20^{\mathrm{NS}}$ & $2.20 \mathrm{ij}$ & $0.40^{\mathrm{NS}}$ & $4.40 \mathrm{kl}$ & $1.20 \mathrm{a}$ & $95.00 \mathrm{~d}$ & $900.0 \mathrm{~m}$ & $121.0 \mathrm{e}$ \\
\hline $0.40^{\mathrm{NS}}$ & $2.90 \mathrm{ab}$ & $0.60^{\mathrm{NS}}$ & $4.80 \mathrm{hi}$ & $1.00 \mathrm{bc}$ & $89.00 \mathrm{~g}$ & 980.01 & $114.0 \mathrm{~h}$ \\
\hline $0.30^{\mathrm{NS}}$ & $2.30 \mathrm{hi}$ & $0.50^{\text {NS }}$ & $5.10 \mathrm{efg}$ & $0.90 \mathrm{~cd}$ & 94.00 de & $1450.0 \mathrm{~g}$ & $121.0 \mathrm{e}$ \\
\hline $0.35^{\mathrm{NS}}$ & $2.57 \mathrm{de}$ & $0.50^{\mathrm{NS}}$ & $5.20 \mathrm{def}$ & $1.00 \mathrm{bc}$ & 98.00 c & $1050.0 \mathrm{k}$ & $127.0 \mathrm{~d}$ \\
\hline $0.40^{\mathrm{NS}}$ & 2.80 bc & $0.60^{\mathrm{NS}}$ & $4.70 \mathrm{ij}$ & $1.10 \mathrm{ab}$ & $95.00 \mathrm{~d}$ & $1820.0 \mathrm{~d}$ & $122.0 \mathrm{e}$ \\
\hline $0.30^{\text {NS }}$ & $2.40 \mathrm{fgh}$ & $0.60^{\mathrm{NS}}$ & 4.90 ghi & $1.10 \mathrm{ab}$ & $86.00 \mathrm{~h}$ & $1550.0 \mathrm{e}$ & $111.0 \mathrm{i}$ \\
\hline $0.30^{\mathrm{NS}}$ & $2.23 \mathrm{ij}$ & $0.50^{\text {NS }}$ & $5.00 \mathrm{fgh}$ & $1.10 \mathrm{ab}$ & $96.00 \mathrm{~d}$ & 750.0 о & $122.0 \mathrm{e}$ \\
\hline $0.20^{\mathrm{NS}}$ & $2.47 \mathrm{efg}$ & $0.50^{\text {NS }}$ & 5.50 bc & $1.10 \mathrm{ab}$ & $85.00 \mathrm{~h}$ & 678.0 p & $111.0 \mathrm{I}$ \\
\hline $0.30^{\mathrm{NS}}$ & $2.70 \mathrm{~cd}$ & $0.70^{\text {NS }}$ & $6.00 \mathrm{a}$ & $1.20 \mathrm{a}$ & $92.00 \mathrm{ef}$ & 1000.01 & $117.0 \mathrm{~g}$ \\
\hline $0.30^{\mathrm{NS}}$ & $2.00 \mathrm{kl}$ & $0.70^{\mathrm{NS}}$ & $6.00 \mathrm{a}$ & $1.00 \mathrm{bc}$ & $72.33 \mathrm{k}$ & $600.0 \mathrm{q}$ & $98.00 \mathrm{~m}$ \\
\hline $0.23^{\mathrm{NS}}$ & 2.50 ef & $0.43^{\mathrm{NS}}$ & $4.70 \mathrm{ij}$ & $1.10 \mathrm{ab}$ & $95.00 \mathrm{~d}$ & $1500.0 \mathrm{f}$ & $121.0 \mathrm{e}$ \\
\hline $0.35^{\mathrm{NS}}$ & 2.43 efgh & $0.60^{\mathrm{NS}}$ & $5.00 \mathrm{fgh}$ & $1.00 \mathrm{bc}$ & $95.00 \mathrm{~d}$ & $550.0 \mathrm{r}$ & $122.0 \mathrm{e}$ \\
\hline $0.20^{\mathrm{NS}}$ & $2.30 \mathrm{hi}$ & $0.40^{\text {NS }}$ & 4.201 & $0.90 \mathrm{~cd}$ & $83.00 \mathrm{i}$ & $1100.0 \mathrm{j}$ & $107.0 \mathrm{l}$ \\
\hline $0.26^{\mathrm{NS}}$ & 2.33 ghi & $0.50^{\mathrm{NS}}$ & $5.00 \mathrm{fgh}$ & $1.00 \mathrm{bc}$ & $99.00 \mathrm{bc}$ & $500.0 \mathrm{~s}$ & 129.0 c \\
\hline $0.22^{\text {NS }}$ & $2.10 \mathrm{jk}$ & $0.40^{\mathrm{NS}}$ & $5.00 \mathrm{fgh}$ & 0.70 ef & $104.0 \mathrm{a}$ & $690.0 \mathrm{p}$ & $133.0 \mathrm{~b}$ \\
\hline $0.20^{\mathrm{NS}}$ & $1.90 \mathrm{~lm}$ & $0.42^{\text {NS }}$ & $4.30 \mathrm{kl}$ & 0.80 de & 94.00 de & $1900.0 \mathrm{c}$ & $122.0 \mathrm{e}$ \\
\hline $0.20^{\mathrm{NS}}$ & $2.20 \mathrm{ij}$ & $0.40^{\mathrm{NS}}$ & $4.50 \mathrm{jk}$ & $0.60 \mathrm{f}$ & $105.0 \mathrm{a}$ & $1350.0 \mathrm{~h}$ & 135.0 a \\
\hline $0.20^{\mathrm{NS}}$ & $2.47 \mathrm{efg}$ & $0.52^{\text {NS }}$ & $4.80 \mathrm{hi}$ & $1.00 \mathrm{bc}$ & 100.0 bc & $1270.0 \mathrm{i}$ & $127.0 \mathrm{~d}$ \\
\hline
\end{tabular}

Values with same letter(s) are statistically identical at $5 \%$ level of probability.

The highest anther length was obtained from D.ShanB and the lowest from IR58025B (Tables 2 and 3). Anther length showed significant variation among the restorer lines (Table 1) and ranged from $1.9 \mathrm{~mm}$ to $3 \mathrm{~mm}$. The maximum anther length was observed in BR168R (3 mm) along with Wan3R and the lowest in IR72906-24-1-3-IR (1.9 mm) (Table 4). Reddy et al. (1997) reported $1.5 \mathrm{~mm}$ to $3 \mathrm{~mm}$ long anther in rice. Anther breadth did not vary significantly among the restorer lines. In contrast, maintainer and CMS lines had statistically significant variation for anther breadth. Anther length and breadth are the important components that attributed to proper pollen quantity and pollen shedding for pollination on CMS lines (Parmar et al. 1979). Filament length is a desirable trait for pollination as it enhances the chance of anther extrusion. Filament length varied significantly among the tested CMS lines, maintainer and restorer lines. The highest was recorded in BRRI9A (5 mm) and the lowest in IR78362A (3 mm). All the maintainer lines showed longer filament length as compared to their corresponding CMS lines except for D.ShanB. 
Filament length ranged from $4 \mathrm{~mm}$ to $5.5 \mathrm{~mm}$ with a mean value of $4.64 \mathrm{~mm}$ (Tables 2 and 3). Filament length varied significantly among the tested restorer lines and it ranged from 4.20 $\mathrm{mm}$ to $6 \mathrm{~mm}$ with a mean value of $5.09 \mathrm{~mm}$. The highest length was recorded in IR73885-1-4-14-4-3-6R and IR69716-37-1-1-5-IR (6 mm) and the lowest was recorded in IR44675R (4.2 mm) (Table 4). Length of style is very important trait in restorer lines as it helps pushing out feathery stigma from the floret. Style length had non-significant variation among CMS and maintainer lines but it exhibited significant variation in restorer lines (Table 1). The per cent panicle exertion ranged from 59.93 to 77.22 . The panicle exertion was recorded maximum in BRRI9A (77.22\%) and the minimum in IR58025A (59.93\%). The panicle exertion directly influences natural out crossing and ultimately resulting into good seed setting (Ramesha et al. 1999). Well-exerted panicles were also advocated for higher seed set by many authors (Taillebois and Guimmareas 1986 and Vinod et al. 1990).

The BRRI developed CMS line BRRI9A was found to have high panicle exertion rate as compared to other CMS lines tested in this study. Stigma exertion rate varied significantly among the CMS lines. Stigma exertion rate was found maximum in IR78362A $(75.67 \%)$ followed by BRRI9A (74.51\%) and the lowest was found in II32A (51.30\%). Kato and Namani (1978) reported that the CMS lines having better stigma exertion had significantly higher seed set than that of spikelets without exerted stigma. Rice is an autogamous crop; high out crossing does not generally occur. Natural out crossing rate of these tested CMS lines ranged from 1.06 to 3.33\% (Table 2). Wide ranges of natural out crossing (10.44\%) were reported in male sterile lines of rice (Azzini and Rutger 1982, Stansel and Craigmiles 1966 and Athwal and Virmani 1972). Maintainer and restorer lines are inbred line and it has natural full exerted panicle and self pollination. So, panicle exertion, stigma exertion and out crossing rate was not taken. The CMS lines had their specific flowering patterns.

The flowering time delayed for all tested CMS lines than that of their respective maintainers. Days to $50 \%$ flowering ranged from 69 to 95 days. The CMS line D.ShanA was found earliest in days to 50\% flowering (69 days) while the CMS line Gan46A was late (95 days) (Table 3). Variation for days to $50 \%$ flowering was found significant among the maintainer lines. In general, maintainer lines flowered earlier than their respective CMS lines. It ranged from 66 to 90 days. Gan46B was late in days to 50\% flowering while D.ShanB was the earliest to flower (Table 3). The difference in floral morphology between CMS lines and their corresponding maintainers was reported by Tomar and Anbalagan (2004). Days to 50\% flowering varied significantly among restorer lines. The earliest flowering time was recorded in IR69716-37-1-1-5-IR (72.33 days) and late in IR69702-3-2-3R (105 days) (Table 4). Chakraborty and Hajarika (1994) reported significant variation among rice genotypes for days to flowering. Days to maturity showed significant variation among the tested CMS lines. Gan46A (121 days) was late maturing followed by BRRI9A (115 days) and D.ShanA (97 days) was earliest.

Days to maturity in the parental lines are the main selection criteria for breeding early maturing hybrids. In respect of days to maturity in the maintainer lines ranged from 94 to 119 days. The earliest line was D.ShanB and the late was Gan46B (Table 3). Days to maturity differed significantly among the restorer lines. It ranged from 98 to 135 days with mean value of 118.90 . The lowest days to maturity was recorded in IR69716-37-1-1-5-IR (98 days) and the highest in IR69702-3-2-3R (135 days). No. of pollen/microscopic focus was found significant among the tested restorer lines. It ranged from 300-2150 pollen/focus (Table 4) with a mean value of 1208.67 pollen/ microscopic focus. Maximum pollen/ microscopic focus was obtained from BR6723-1-12R followed by BR168R and BR6839-41-5-1R and the minimum was observed in M.H.63R. BRRI developed restorer line BR6723-1-1-2R, BR168R and BR6839-41-5-1R found to have high pollen loads as compared to other restorer lines. 
Floral characteristics of the component lines revealed that CMS line BRRI9A and its corresponding maintainer line BRRI9B possessed excellent floral characteristics that could influence out crossing potentiality if restorer line BR168R is used for hybrid seed production.

\section{References}

Amirjani MR. 2011. Effect of salinity stress on growth, sugar content, pigments and enzyme activity of rice. Int. J. Bot. 7(1): 73-81.

Athwal DS and Virmani SS 1972. Cytoplasmic male sterility and hybrid breeding in rice. Pages 615-620 in Rice Breeding. IRRI. PO Box 933, Manila, Philippines.

Azzini LE and Rutger N 1982. Amount of out crossing on different CMS lines of Rice. Crop. Sci. 22: 905906.

Chakraborty S and Hajarika MH 1994. Estimation of various genetic parameters of yield and yield components of rice. Oryza 31: 226-227.

Huda N and Shahjahan Ali 2012. Hybrid rice seed technology in Bangladesh. Issues and Considerations. Seminar presented at BARC, Farm gate, Dhaka on September 18, 2012.

Ifterkharuddaula KM, Islam MR, Aditya TL, Biswas JK and Bashar MK 2011. Development of climate change resilient rice varieties: current status and potential. Souvenir, SAARC seed congress \& fair 2011, held at Bangabandhu International Conference Centre during 10-12 April, 2011, Dhaka, Bangladesh.

Kato $\mathrm{H}$ and Namani $\mathrm{H}$ 1978. Floral characteristics and environmental factors increasing natural out crossing rate for $\mathrm{F}_{1}$ seed production in rice (Oryza sativa L.) Jpn. J. Breed. 37: 318-330.

Oka HI 1988. Origin of cultivated rice. Jpn. Sci. Press, elesvier, Tokyo, pp. 254.

Parmar KS, Siddiq EA and Swaminathan MS 1979. Variation in components of flowering behavior of rice. Indian J. Genet. 39: 542-550.

Ramesha MA, Virakmath BC, Ahamad IIyas M. and Vijaykumar CHM 1999. New CMS sources with stable male sterility and better out crossing traits in rice. Indian J.Genet. 59: 403-409.

Reddy JN, Pani D and Roy JK 1997. Variability and character association in low land rice. Oryza. 23(4): 2125.

Seed Certification Agency (SCA) 2012. List of released hybrid rice varieties from 1999 to 2011.

Stansel JW and Craigmiles JP 1966. Hybrid rice problems and potentials. Rice J. 69: 14-15, 46.

Taillebois J and Guimmareas EP 1986. Improving out crossing rate in rice (Oryza sativa L.). In: Hybrid Rice. Proc. Intern. Symp. on Hybrid Rice, 6-10 October, 1986. Changsha, Hunan, China. International Rice Research Institute, Los Banos, Philippines.

Tomar SMS and Anbalagan S 2004. Characterization of cytoplasmic male sterile line in wheat (Triticum aestivum L.). Ind. J.Genet. 64(3): 189-195.

Vinod KK, Vivekanadan P and Subrahmanian M 1990. Effect of cytoplasmic male sterility on panicle exertion and sheath rot incidence in $\mathrm{F}_{2}$ rice hybrids. Int. Rice Res. Newsl. 15(2): 5.

Virmani SS and Edwards IB 1983. Current status and future prospects for breeding rice and wheat. Adv. Agron. 36: 184-186. 